\title{
Influence of rotating speed and trial weight on one-time balancing Xueshan Xu ${ }^{1,}$, Yujie Bai ${ }^{1, b}$, Xiao-Zhang Zhang ${ }^{1, c^{*}}$ \\ ${ }^{1}$ Department of Engineering Physics, Tsinghua University, Beijing 100084, P.R.China

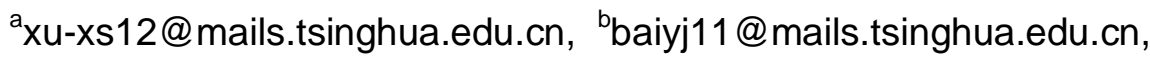 'zhangxzh@mail.tsinghua.edu.cn
}

Keywords: dynamic balance, measurement error, trial weight and angle, balancing speed, one time balancing effect

Abstract. In practical process of dynamic balancing, rotating speed, mass and angular location of trial weight will affect one-time balancing effect. In order to find the best balancing speed, trial weight mass and angle, computer simulation and experimental research are conducted. The results show that, one can obtain a better balancing effect when the ratio of trial weight and equivalent original unbalance mass is between 1.5 and 2, and the angle difference between trial weight and original unbalance is about $140^{\circ} \sim 180^{\circ}$.

\section{Introduction}

For single plane dynamic balance, it can be proved that the influence coefficient method works well. Theoretically, the balance error by this method can be very small to be ignored. But in actual operation process, one time balancing effect is still limited. Multiple steps of balance are often required. Due to speed fluctuation [1], signal noise, mechanical and electrical oscillating etc, the measured vibration signals contain errors in balancing process. This further causes calculated balancing mass and angle errors, which influence the balancing effect.

In this paper, these factors of influence were investigated in details by theoretical analysis and by experimental test. The purpose is to find more practical ways for the dynamic balance, and to improve the efficiency of one-time balancing.

\section{Theoretical study}

In this section, theoretical study of influence of size and angle of trial weight on one time balancing effect is presented. The method of influence coefficient requires one to measure two parameters: original vibration signal $\overrightarrow{R_{0}}$ without a trial weight and vibration signal $\overrightarrow{R_{1}}$ with the trial weight. From the principle of influence coefficient method, we know that the calculated unbalance mass is the same as long as the angle and module differences of $\overrightarrow{R_{0}}$ and $\overrightarrow{R_{1}}$ are unchanged. So the measurement errors of $\overrightarrow{R_{0}}$ and $\overrightarrow{R_{1}}$ can be summarized to the errors of the angle and module differences of $\overrightarrow{R_{1}}$.

Then one can define an $\overrightarrow{R_{1}^{r}}$, which has variations $\Delta \mathrm{R}_{1}$ and $\Delta \theta$ by assuming $\overrightarrow{R_{0}}$ and $\overrightarrow{R_{1}}$ without errors. $\overrightarrow{\boldsymbol{R}_{1}^{\prime}}$ and $\overrightarrow{\boldsymbol{R}_{1}}$ have the following relation:

$$
\stackrel{\mathbf{m}}{R_{1}^{\prime}}=\left(R_{1}+\Delta R_{1}\right) \exp (\theta+\Delta \theta),
$$

While the influence coefficient method tells us the relation between equivalent original unbalance mass $\overrightarrow{m_{0}}$ and trial weight $\overrightarrow{m_{1}}$ is:

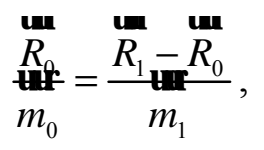

When calculating with $\overrightarrow{R_{1}^{z}}$, the equivalent original unbalance mass is named by $\overrightarrow{m_{x}}$, and 


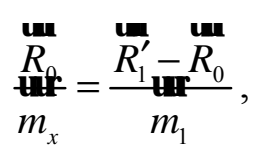

From Eq.2 and Eq.3, we have:

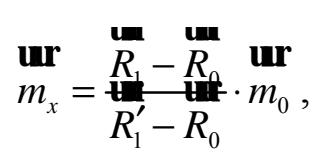

Owing to the measurement errors of $\Delta \mathrm{R}_{1}$ and $\Delta \theta$, the residual equivalent unbalance mass $m_{0}^{\prime}$ after one-time balancing is as follows:

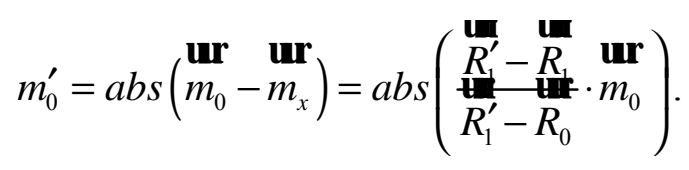

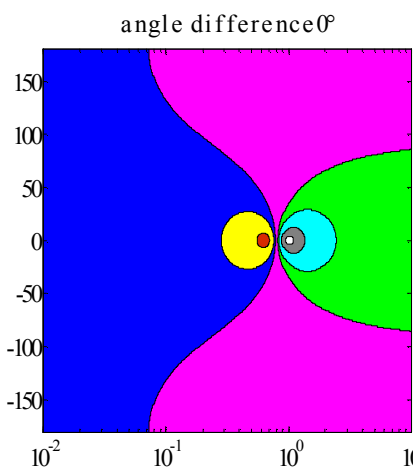

angle difference $67.5^{\circ}$

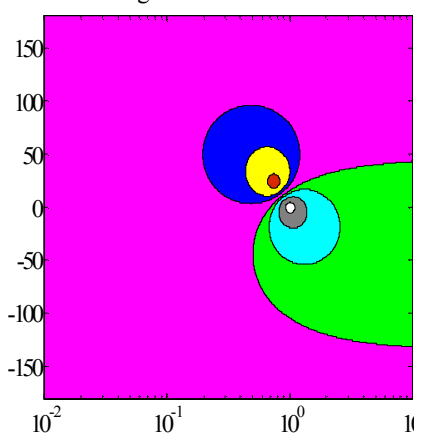

angle difference $135^{\circ}$

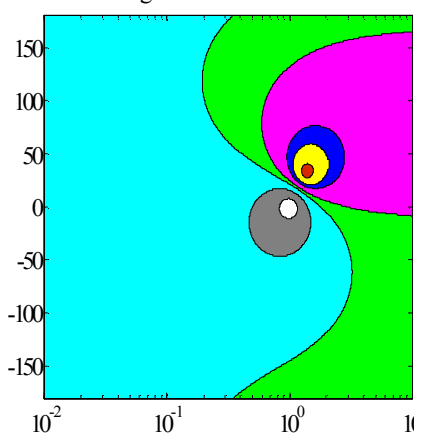

angle difference $22.5^{\circ}$

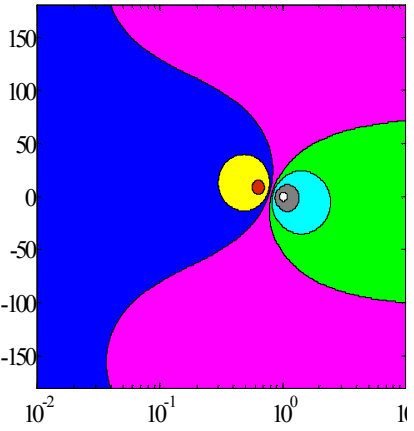

angle difference $90^{\circ}$

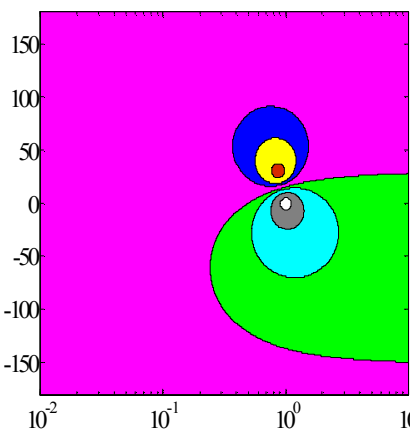

angle difference $157.5^{\circ}$

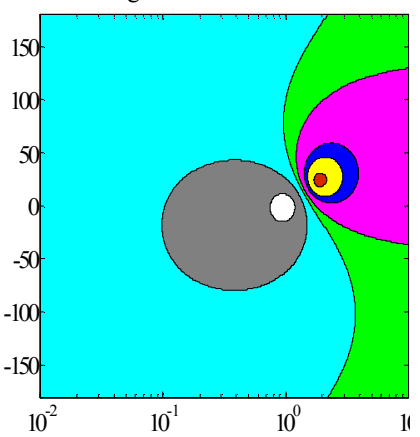

angle difference $45^{\circ}$

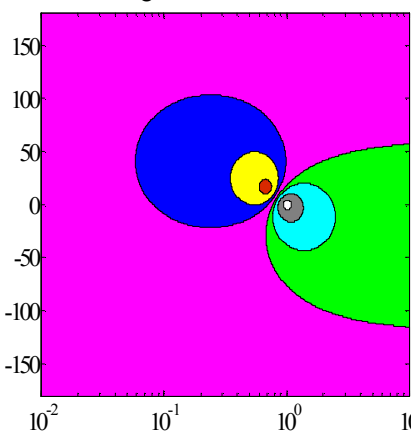

angle difference $1125^{\circ}$

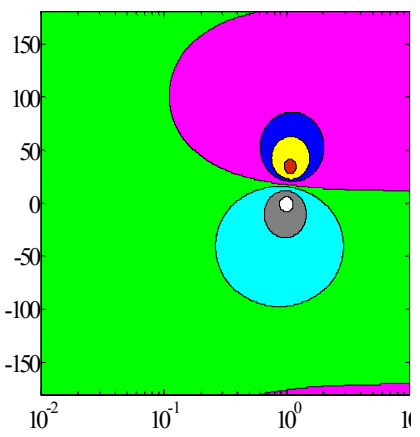

angle difference $180^{\circ}$

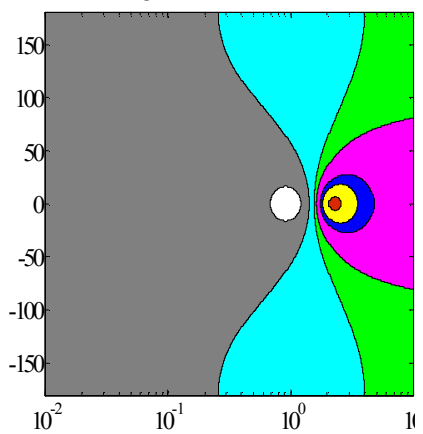

Fig 1 One time balancing effect diagrams under different relative size and angle errors (the ratio of $m_{1} / m_{0}$ is 0.557 )

The influence of $\Delta \mathrm{R} 1$ and $\Delta \theta$ on one-time balancing is simulated with MATLAB. Results are shown in Fig 1. In each of the following diagrams, x-axis is the relative module error $\left|\overrightarrow{R_{1}^{\prime}}\right| /\left|\overrightarrow{R_{1}}\right|$; 
$y$-axis is the angle error $\Delta \theta$. Different colors represent different value ranges of $m_{0}^{\prime} / m_{0}$. These are listed in Table 1.

Table1 The colors presenting different values of $m_{0}^{\prime} / m_{0}$ in Fig 1

\begin{tabular}{ccccc}
\hline color & white & gray & light blue & green \\
\hline value range & {$[0,0.2)$} & $(0.2,0.5)$ & $(0.5,0.8)$ & $(0.8,1)$ \\
\hline color & magenta & blue & yellow & red \\
\hline value range & $(1,1.5)$ & $(1.5,2)$ & $(2,5)$ & $(5,+\infty)$ \\
\hline
\end{tabular}

From Fig 1 we can see that, for a given value of trial weight, the effective balance region $\left(m_{0}^{r} / m_{0}\right.$ $<1)$ consisting of the white, gray, light blue, and green region becomes larger as the angle difference between the trial weight and the original imbalance. At the same time, the invalid balance region $\left(m_{0}^{r} / m_{0}>1\right)$ consisting of the magenta, blue, yellow, and red region becomes smaller. Therefore, the balancing effect is better if the angle difference is larger (proper $>140^{\circ}$ ).

We tried to change the ratio of $m_{1} / m_{0}$, and found that there are similar rules as those in Fig 1 under different mass ratio. In order to study the influence of trial weight mass on one-time balancing effect, diagrams were drawn as shown in Fig 2 for different ratios of $m_{1} / m_{0}$, setting the angle difference between trial weight and original unbalance as $160^{\circ}$.

$\mathrm{m} 1 / \mathrm{m} 0=0.2$

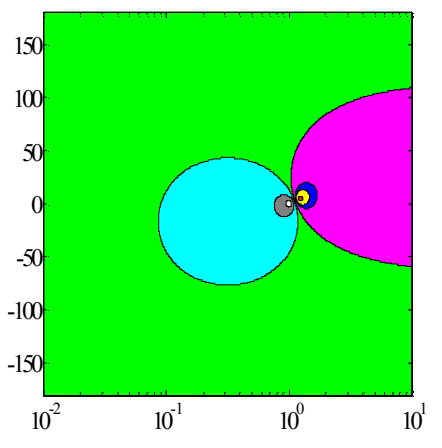

$\mathrm{ml} / \mathrm{m} 0=1$

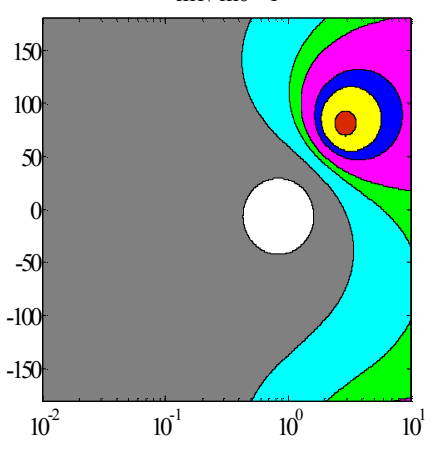

$\mathrm{ml} / \mathrm{m} 0=2$

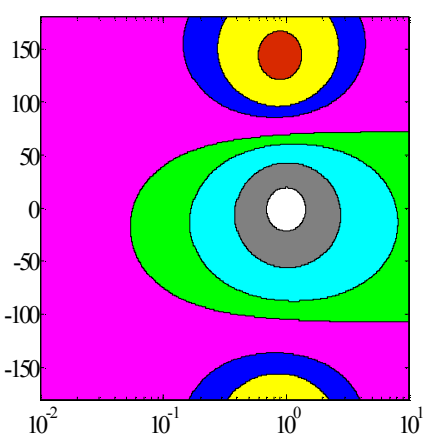

$\mathrm{m} 1 / \mathrm{m} 0=0.5$

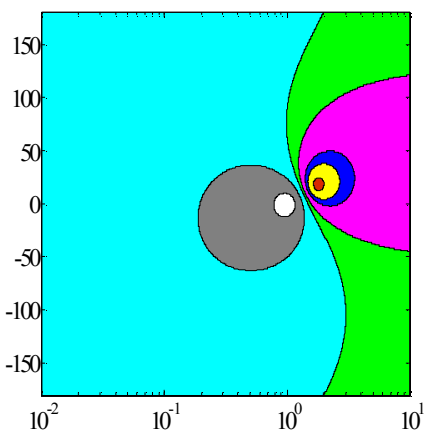

$\mathrm{m} 1 / \mathrm{m} 0=1.2$

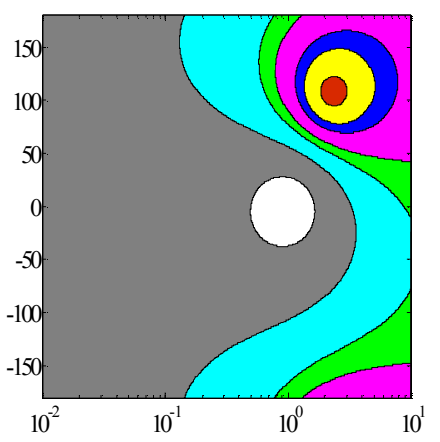

$\mathrm{m} 1 / \mathrm{m} 0=5$

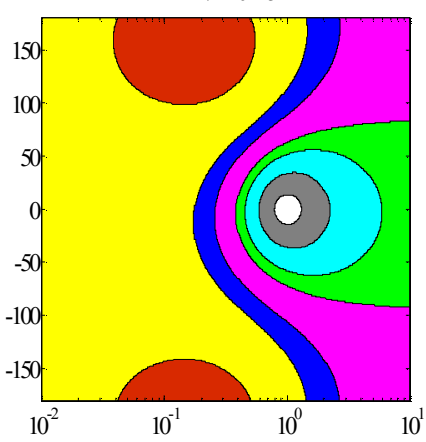

$\mathrm{m} 1 / \mathrm{m} 0=0.8$

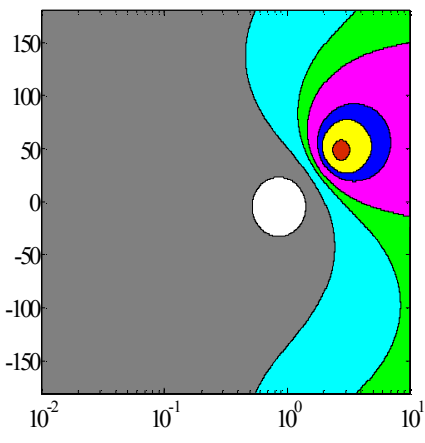

$\mathrm{m} 1 / \mathrm{m} 0=1.5$

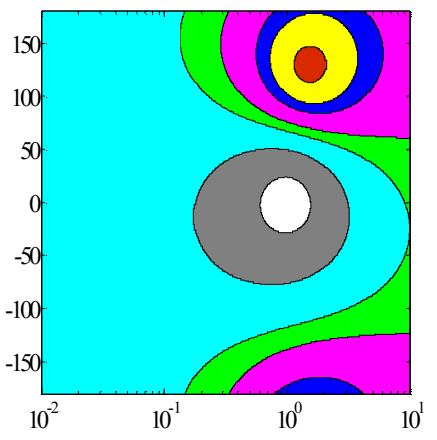

$\mathrm{m} 1 / \mathrm{m} 0=10$

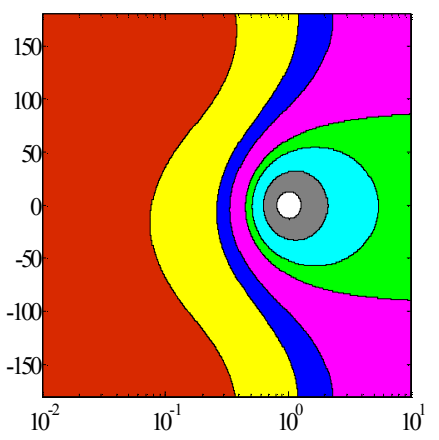

Fig 2 one time balancing effect diagrams under different ratios of $m_{1}$ and $m_{0}$ (the angle difference between $m_{1}$ and $m_{0}$ is $160^{\circ}$ ) 
From Fig 2 we know that, when $m_{1} / m_{0}<1$, the effective balance region is similar in size. With mass ratio $m_{1} / m_{0}$ increasing, the balancing effect is getting better and better within the effective balancing area (under the same deviation, the remaining unbalance becomes smaller). The most obvious manifestation is that the white circle area is larger and larger. When $m_{1} / m_{0}>1$, with the quality ratio increases, the effective balance region is getting smaller and smaller. On the contrary, invalid region becomes larger. Within the effective region, the balancing effect becomes worse and worse. Thus, when selecting the weight, the optimum ratio $\mathrm{m}_{1} / \mathrm{m}_{0}$ is between $0.5 \sim 2$.

\section{Experimental analysis}

The Dong Hao rotor experimental platform was used to study the influences of balancing speed, trial weight mass and trial weight angle. Sensor is Bentley eddy current displacement sensor. A data acquisition card is NI data acquisition card PCI6221. The rotor rotation controller of test DH5600 is made by Dong Hao. Schematic diagram of the rotor test rig is shown in Fig 3. The computer analysis program coding with Labview uses digital correlation filtering method [2] to process signals.

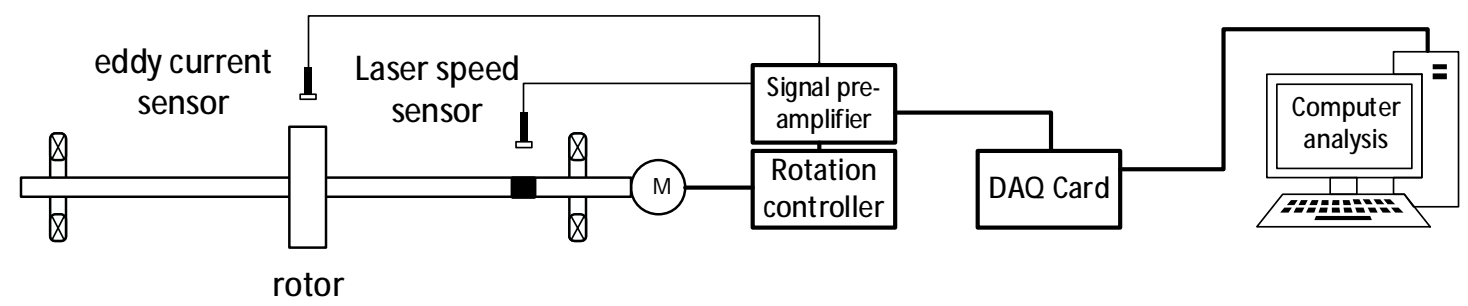

Fig 3 Schematic diagram of rotor test rig

In the experiment, the rotor was balanced at $20 \mathrm{~Hz}, 30 \mathrm{~Hz}, 40 \mathrm{~Hz}, 50 \mathrm{~Hz}, 60 \mathrm{~Hz}, 70 \mathrm{~Hz}, 80 \mathrm{~Hz}$ and $90 \mathrm{~Hz}$ (the resonance frequency of the rotor system is $55 \mathrm{~Hz}$ ). The original equivalent unbalance is $0.395 \mathrm{~g} \angle 31.6^{\circ}$. Trial weights were $0.22 \mathrm{~g}, 0.53 \mathrm{~g}, 0.71 \mathrm{~g}$ and $0.79 \mathrm{~g}$ respectively, and their angular locations were $45^{\circ}, 67.5^{\circ}, 90^{\circ}, 112.5^{\circ}, 135^{\circ}, 157.5^{\circ}, 180^{\circ}$ and $202.5^{\circ}$ respectively. Experimental results are shown in Fig 4.

In Fig 4, when the balancing speed is at the one from $20 \mathrm{~Hz}$ to $60 \mathrm{~Hz}$, the value of $m_{0}^{\prime} / m_{0}$ is obviously larger than the value of the one from $70 \mathrm{~Hz}$ to $90 \mathrm{~Hz}$. Overall, the value of $m_{0}^{z} / m_{0}$ decreases with the increase of the angle difference between trial weight and the original unbalance. When the ratio of $\mathrm{m}_{1} / \mathrm{m}_{0}$ is 1.798 or 2.004 , the ratio of $m_{0}^{l} / m_{0}$ is relatively smaller than that when $\mathrm{m}_{1} / \mathrm{m}_{0}$ is 0.557 or 1.342 . When the ratio $\mathrm{m} 1 / \mathrm{m} 0$ or the angle difference between trial weight and original unbalance changes, the change of $m_{0}^{i} / m_{0}$ is not obvious. But when balancing speed changes, the value of $m_{0}^{\prime} / m_{0}$ changes greatly.

From Fig 4 we can see:

(1) The influence on one-time balancing result caused by the balancing speed is greater than that caused by trial weight size and angle. In the supercritical region of $70 \mathrm{~Hz}, 80 \mathrm{~Hz}$ and $90 \mathrm{~Hz}$, the balancing effects are better, compared with the effects in resonance region and sub critical region.

(2) Overall, when the trial weight is large ( the ratio of $\mathrm{m} 1 / \mathrm{m} 0$ is about 2 ) and the angle difference between trial weight and original unbalance is large $\left(>140^{\circ}\right)$, the balancing effect is better.

Experimentally measured amplitude-frequency curve is shown in Fig $5(\mathrm{Up})$. As can be seen from Fig 5 , in the low speed region, the amplitude of the rotor is not close to 0 . This is because the measured shaft section is of mechanical or electrical runout, for examples, permanent bending, surface roundness and local defects of shaft, local residual magnetism, and so on. One way of eliminating this effect is slow rolling compensation [3]. When the rotor speed is very slow, the vibration caused by imbalance is very small. It can be assumed that the vibration is caused by mechanical or electrical runout only. Therefore, the vibration signal in the slow rolling state can be used to compensate measuring signals at higher speeds. The accuracy of the compensation has great influence on subcritical vibration signals, and has less influence on critical and supercritical signals. 
So the balancing effect in the subcritical region is relatively poor. Amplitude-frequency curve with compensation is shown in Fig 5 (Down).
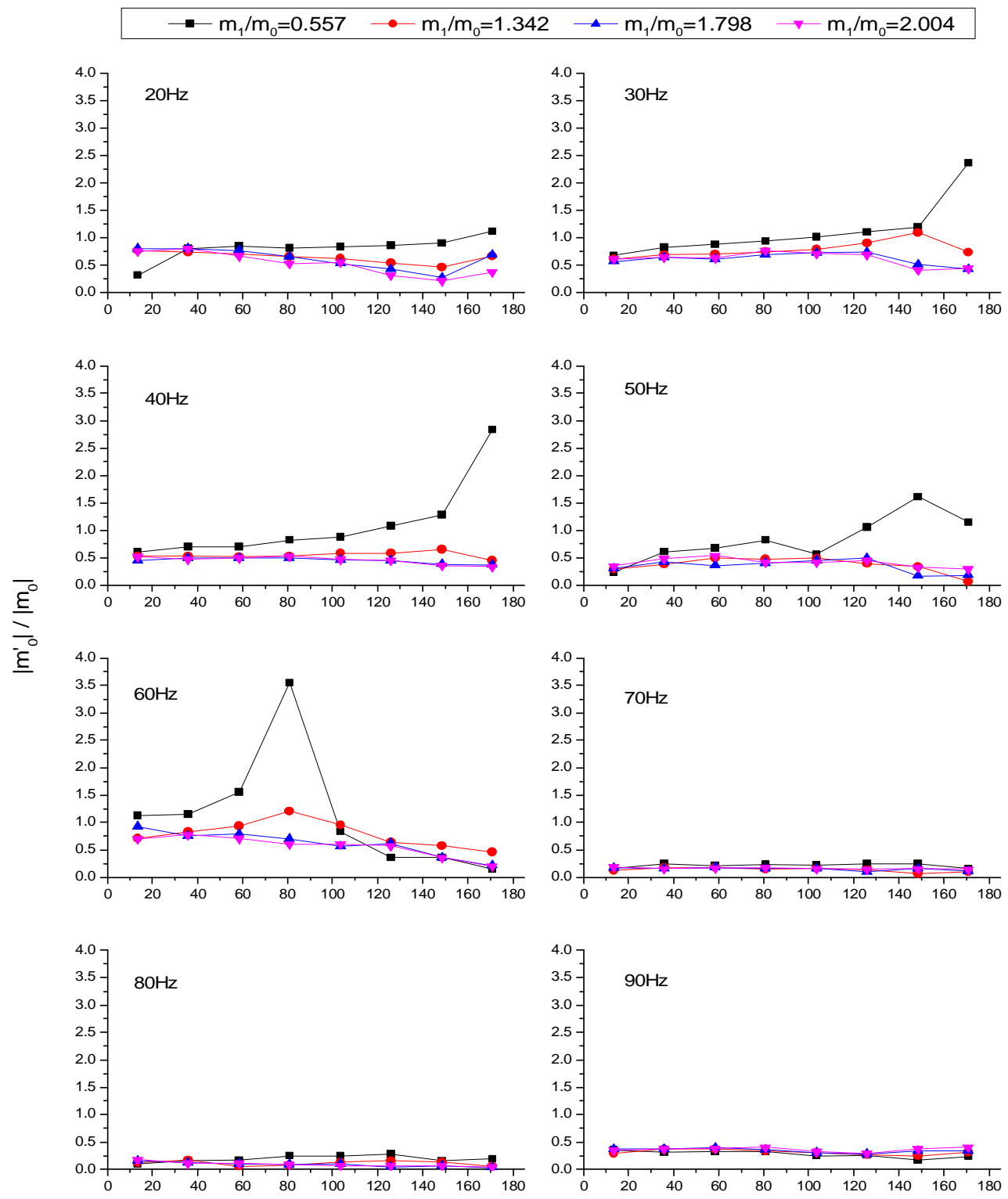

The angle difference between $m_{1}$ and $m_{0}$

Fig 4 The influence of mass and angle of trial weight on one time balancing effect under different rotating speeds

In the resonance region, the stability of the rotor's rotation is poor. The errors of amplitude and phase are relative large. Compared to the supercritical region, the balancing effect in the resonance region is relatively poor. In the supercritical region, due to the automatic centering, the amplitude and phase are relatively stable and the measurement error is relatively small. Therefore the balancing effect in this region is better.

From Fig 2, the optimum ratio of $\mathrm{m} 1 / \mathrm{m} 0$ is between $0.5 \sim 2$. But when $\mathrm{ml} / \mathrm{m} 0$ is $0.5 \sim 1.5$, it is unwise to make the angle difference between $\mathrm{m} 1$ and $\mathrm{m} 0$ large. Because the vibration signal with the trial weight will become small, making the measurement error large. This is shown in Fig 4. So the optimal ratio $\mathrm{m} 1 / \mathrm{m} 0$ is about $1.5 \sim 2$. 


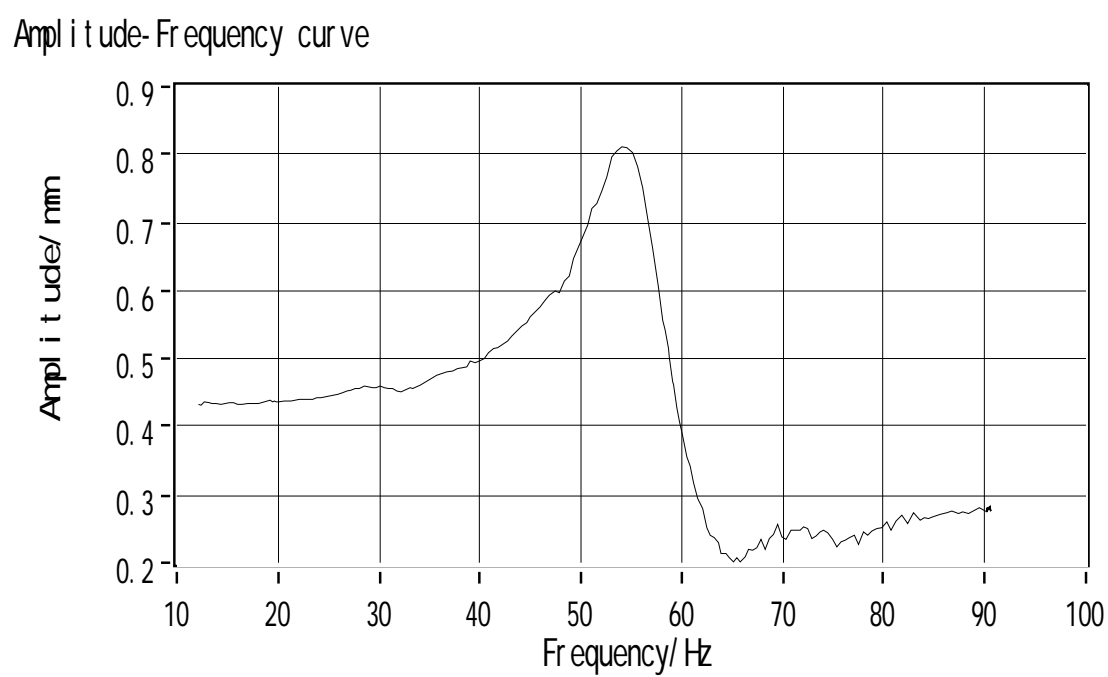

Ampl i tude- Frequency curve

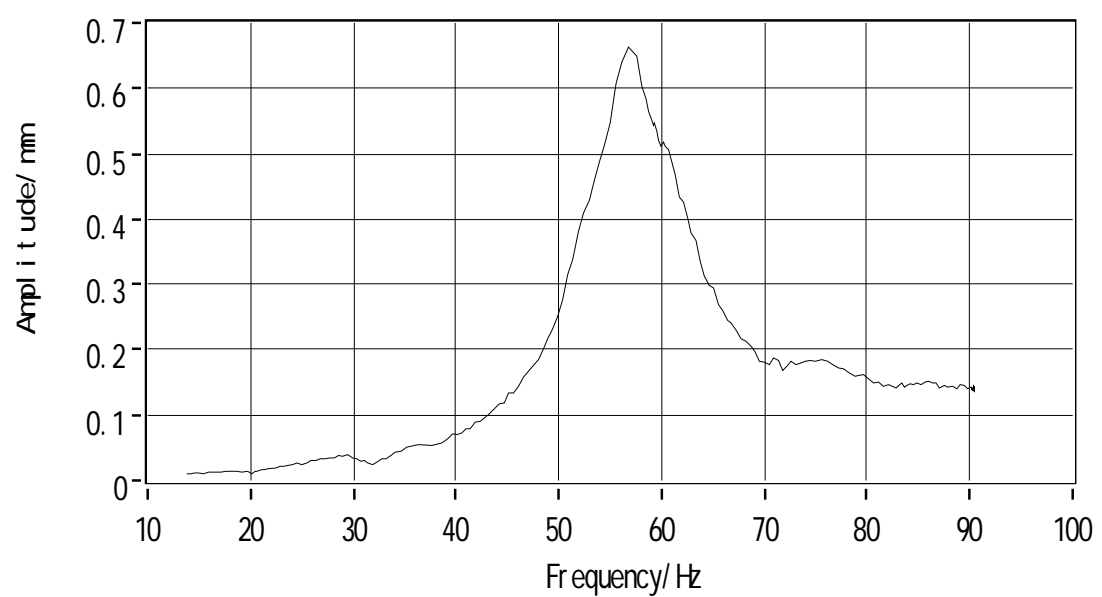

Fig 5 Amplitude frequency curve without compensation (Up) and amplitude frequency curve with compensation (Down)

\section{Conclusions}

In summary, the following conclusions can be drawn:

(1) Considering the one-time balancing effect and the rotor system safety, the optimal ratio of trial weight and the original equivalent unbalance mass is between 1.5 and 2 , and the optimal angle difference between the trial weight and original unbalance is about $140^{\circ} \sim 180^{\circ}$.

(2) From the viewpoint of balancing effect, it is better to balance the rotor in the supercritical region.

\section{References}

[1] Fangze Li, Fuqing Liu, Zheng Wang, Engineering Vibration Test and Analysis, first ed., Higher Education Press, Beijing, 1992.(In Chinese)

[2] Hongyu Shu, Lingfei Xie, Yidong Zhang, et al, Application of Digital Correlation Filtering in Rotor-balance Measuring Theory, Journal of Chongqing University(Natural Science Edition), 2005,vol.28, no.9, pp. 27-30. (In Chinese)

[3] Donald E Bently, Charles T. Hatch, (Hongliang Yao Trans.), Fundamentals of Rotating Machinery Diagnostics, China Machine Press, Beijing, 2014. (In Chinese) 